\title{
The effect of edaravone to leukocyte rolling and adhesion molecule expression in microvessels
}

\author{
Kumi Sakamoto $^{1}$, Tsutomu Yamashita ${ }^{2 *}$, Hiroshi Yamanishi ${ }^{2}$, Junichiro Yamamoto $^{1}$ \\ ${ }^{1}$ Laboratory of Physiology, Faculty of Nutrition, Kobe Gakuin University, Kobe, Japan \\ ${ }^{2}$ Laboratory of Medical Technology, Faculty of Nutrition, Kobe Gakuin University, Kobe, Japan; \\ *Corresponding Author: tsutomu@nutr.kobegakuin.ac.jp
}

Received 19 October 2012; revised 22 November 2012; accepted 19 January 2013

\section{ABSTRACT}

Background: Recently, it has become apparent that reactive oxygen species (ROS) play a critical role in the initiation of atherosclerosis. In this study, the effect of radical scavenger edaravone to the leukocyte rolling and to the expression of adhesion molecules on microvascular endothelium was investigated. Methods: DSS induced rat colitis model was used as an inflammation model. Edaravone $(10.5 \mathrm{mg} / \mathrm{kg}$, Mitsubishi Tanabe Pharma Corporation, Japan) was used to examine the action of ROS. Images of leukocyte rolling in mesenteric microvessels were investigated in a fluorescence bio-imaging model. Each cross section from the target blood vessel (aotic root, aorta, superior mesenteric artery) were examined by immune-peroxidase staining with anti-P-selectin, E-selectin, ICAM-1 antibody using the streptavidin/biotinylated horseradish peroxidase method. Results: 1) Leukocyte rolling in mesenteric microvessels was significantly increased in colitis. The number of rolling leukocyte was significantly decreased in edaravone group than placebo group (501.3 \pm 39.2 vs $252.2 \pm 37.2$ count $/ 100 \mu \mathrm{m} / 10 \mathrm{~min})$. 2) The expression of P-selectin in endothelial cell was significantly increased in colitis. However, this expression was decreased in edaravone group. 3) The expression of E-selectin was not induced to intact aortic root and aorta. In the superior mesenteric artery, the expression was induced by inflammation, and it was attenuated by edaravone. 4) There was little expression of ICAM-1 in both intact aortic root and aortas. While, in the superior mesenteric artery, the expression was confirmed only in placebo group in colitis, and it was attenuated in edaravone group. Conclusions: It was suggested that administration of edaravone led to improving a haemostasis of microcirculation based on down-regulation of adhesion molecules. These results support the evidence that ROS plays a critical role in microvascular dysfunction.

Keywords: Edaravone; Radical; Scavenger; Atherosclerosis; P-Selectin; E-Selectin; ICAM-1

\section{INTRODUCTION}

Various risk factors concerning the life style related diseases generate reactive oxygen species (ROS). The lipoperoxide production [1,2] and cell damage [3] induced by ROS leads to the formation of arteriosclerotic lesion and microcircular damages. The majority of ROS generated in vivo is extinguished by antioxidant and anti-oxidative enzyme. However, ROS excessively generated oxidizes critical biogenic substances such as DNA, lipid, enzyme and protein [4]. Especially, a lot of reports show that the oxidative damage of such a biogenic substance leads to the crises and development of atherosclerosis [57]. Oxidized LDL in the oxidized biogenic substances is believed to increase especially by enhancement of oxidative stress.

Oxidized LDL contributes to the crises and development of human coronary artery plaque along with the disturbance of vascular endothelial cell and the accumulation of inflammatory cell. In addition, the possibility that this oxidized LDL contributes to the plaque destabilizing is pointed out $[8,9]$. This oxidized LDL is unrestrictedly ingested through the scavenger receptor, and leukocyte, especially monocyte and macrophage becomes foam cells. This is an essence of the arteriosclerotic lesion, and the leukocyte initiates the rolling phenomenon to infiltrate into the subendothelial. Leukocyte tissue invasion is admitted in the formation of basic arteriosclerotic lesion $[10,11]$. Therefore, the leukocyte rolling phenomenon and the leukocyte adhesion are admitted in leukocyte tissue invasion. The cell adhesion moleculesare related to this rolling phenomenon and adhesion. However, in vivo, the interaction between these phe- 
nomena and ROS are not well understood. 3-Methyl-11-phenyl-2-pyrazolin-5-one (edaravone), a free-radical scavenger, has been used clinically in Japan for the treatment of cerebral infarction since 2001, and it has been reported to improve clinical outcomes in patients with ischemic stroke [12-14]. The oxidative stress that occurs after an ischemic stroke produces reactive oxygen species (ROS) like hydrogen peroxide $\left(\mathrm{H}_{2} \mathrm{O}_{2}\right)$, hydroxyl radical $\left(\mathrm{HO}^{\circ}\right)$ and superoxide anion radical $\left(\mathrm{O}_{2}^{--}\right)$that bring about membrane lipid peroxidation and vascular endothelial cell injury $[15,16]$. Edaravone traps a variety of freeradical species including hydroxyl radicals, and is a low molecular weight lipophilic free radical scavenger. The primary focus of edaravone research has revolved around its potential to scavenge hydroxyl, peroxyl and superoxide radicals that mediate neuronal and vascular damage. Experimental studies have revealed that edaravone decreases oxidative stress [17], and its neuroprotective effects are indisputable [18,19].

The present study aimed to examine the influence of edaravone on leukocyte rolling and adhesion molecules expression in vivo.

\section{MATERIAL AND METHODS}

\subsection{Experimental Animals}

Male Wistar-ST rats weighing 250 to $330 \mathrm{~g}$ were obtained from Japan SLC, Inc. (Hamamatsu, Japan). All animals were maintained in air-conditioned rooms (temperature: $22.5^{\circ} \mathrm{C} \pm 0.5^{\circ} \mathrm{C}$; humidity: $50 \% \pm 5 \%$ ) with a 12-h light-dark cycle. Animals had free access to food and drinking water. All procedures were conducted in compliance with the Guiding Principles for the Care and Use of Animals in the Field of Physiological Science of the Physiological Society of Japan.

\subsection{Free-Radical Scavenger}

Edaravone (MCI-186; [3-Methyl-1-1-phenyl-2-pyrazolin-5-one), a free-radical scavenger, was kindly donated by Mitsubishi Pharma Corporation (Tokyo, Japan). Edaravone was administered to rats by infusion into the femoral vein with an infusion pump over a period of 60 $\min \left(10.5 \mathrm{mg} \cdot \mathrm{kg}^{-1} \cdot \mathrm{h}^{-1}\right)$.

\subsection{DSS-Induced Rat Colitis Model}

Three-percent DSS (dextran sulfate sodium salt; Nacalai Tesque, Inc. Japan) in water was orally administered to rats for 5 days after pre-feeding to induce colitis [20]. Oral administration of DSS solution to rodents is widely employed as a model of human ulcerative colitis because it causes acute inflammatory reactions and ulceration along the entire colon that are similar to those observed in human colitis $[21,22]$. This DSS-induced colitis model was used to this study as an inflammatory model.

\subsection{Intravital Imaging of Leukocyte Rolling in the Mesenteric Microcirculation}

Rats were anesthetized by intramuscular injection with pentobarbital $(65 \mathrm{mg} / \mathrm{kg})$ and then used in a bio-imaging model. The bio-imaging system consisted of a biological microscope (Olympus BX51), a confocal scanner unit (CSU-X1; Yokokawa Electric Corporation, Japan), EMCCD camera (Electron multiplying CCD camera YHQ-CascadeII512B; Nippon Roper, Japan), a DPSS (Diode-pumped solid-state ) laser combiner system (Yokokawa Electric Corporation, Japan), and a shutter controller (Yokokawa Electric Corporation). Images were acquired along the $\mathrm{z}$-axis by connecting objects of microscope with a piezo system (Nippon Roper).

The femoral artery and vein were cannulated with a polyethylene catheter. The abdomen was opened via a midline incision, and the ileocecal portion of the mesentery was exposed. The mesentery was continuously perfused at $2.0 \mathrm{~mL} / \mathrm{minwith}$ Krebs-Henseleit buffer saturated with $95 \% \mathrm{~N}_{2} / 5 \% \mathrm{CO}_{2}$ at $2.0 \mathrm{~mL} / \mathrm{min}$ at $37^{\circ} \mathrm{C}$. Blood flow was assessed by measuring red cell velocity by a fiber-optic laser-Doppler anemometer microscope (FLDAM) [23]. Edaravone (10.5 mg/kg/4 mL, edaravone group) or saline (placebo group) was administered to rats through the femoral vein over a period of $60 \mathrm{~min}$ by using an infusion pump.

In the exposed mesenteric microvessels, the target microvessels, which have in $30-35 \mu \mathrm{m}$ diameter and $5.0 \pm$ $0.2 \mathrm{~mm} / \mathrm{sec}$ blood flow velocity, were selected for determination of leukocyte rolling. CFSE $(1 \mathrm{mg} / \mathrm{ml}, 300 \mu \mathrm{l})$ (5- or 6-(N-Succinimidyloxycarbonyl)-fluorescein 3',6'diacetate, Dojindo Molecular Technologies, Inc. Japan) was administered from the right femoral veinover a period of $1 \mathrm{~min}$ at $30 \mathrm{~min}$ after the start of edaravone infusion. CFSE is cell-membrane permeable and readily accumulates inside viable leukocytes where it covalently attaches to intracellular proteins. Hydrolyzed CFSE emits fluorescence and covalently attached fluorescein molecules are kept in the inside of cells. Hydrolyzed CFSE emits fluorescence and covalently attached fluorescein molecules are kept in the inside of cells. Subsequently, the CFSE was excited at a wavelength of $480 \mathrm{~nm}$, and images were collected through a 535/40-nm band-pass emission filter for 10 minutes.

The number of rolling luekocytes was derived from the fluorescence of CSFE-stained luekocytes. Confocal images were captured for ten minutes by the rate of 60 frames per second. The number of rolling luekocytes was counted with image analysis software (Image-Pro, Nippon Roper, Media Cybernetics) by the following ratio, counts/100 $\mu \mathrm{m}$ length/10 minutes. 


\subsection{Immunochemistry}

Immediately after administering of edaravone, anesthetizedrats hearts were exposed by dissection and a wing-shaped needle was inserted into the left ventricle. The blood vessels were then washed thoroughly with buffer (10 mM PBS, pH 7.4) by infusion in situ for approximately $3 \mathrm{~min}$. The target blood vessel areas in each group (aortic root, aorta, superior mesenteric artery) (Figure 1) were excised, fixed with 4\% paraformaldehyde phosphate buffer solution (Wako Pure Chemical Industries Ltd., Japan), soaked in Optimal Cutting Temperature compound (Tissue-Tek and Sakura), and frozen with dry ice and acetone. Six micrometer-thick frozen sections were cut with a cryostat, placed on poly-L-lysine-coated microscope slides (Muto Pure Chemicals, Co., Ltd., Japan), wrapped in aluminum foil, and stored at $-80^{\circ} \mathrm{C}$. Sections were examined by immunoperoxidase staining with anti-P-selectine antibody, anti-E-selectine, anti-ICAM-1, anti-VCAM-1 (Lab Vision Corporation) using the streptavidin-biotinylated horseradish peroxidase method (LSAB2 kit; Dako, Japan) [24].

\subsection{Statistical Analysis}

Results were expressed as mean \pm SEM. Normal distribution was examined, and all data showed the normal distribution. Comparisons among groups were made by using 1-way factorial ANOVA, followed by the Fisher PLSD test, Dunnett's multiple comparison post-test. Differences between means were considered significant at $p$ $<0.05$.

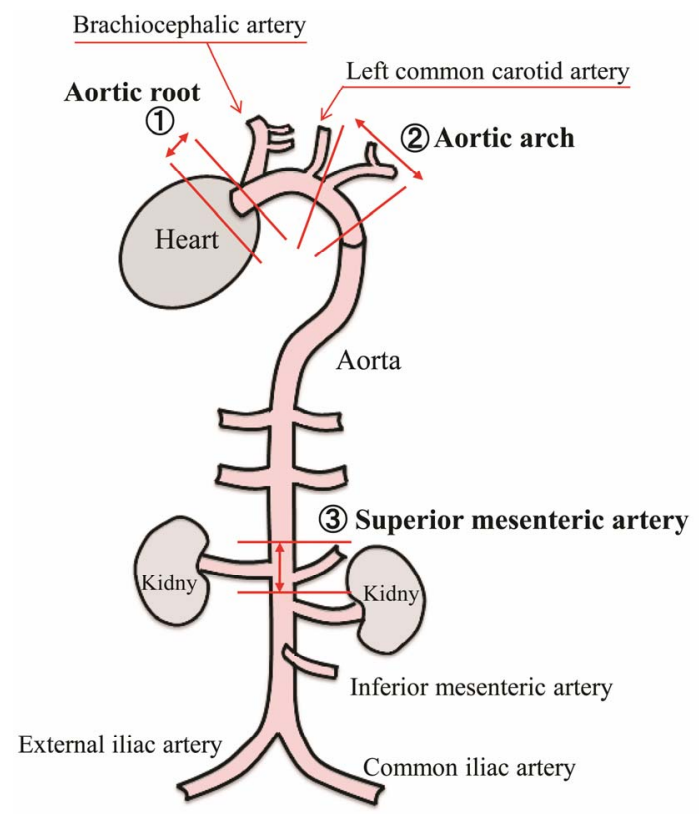

Figure 1. Area for immunohistochemical staining (1) Aortic root; (2) Aorta; (3) Superior mesenteric artery.

\section{RESULTS}

\subsection{Imaging of Leukocyte Rolling in DSS Induced Colitis Model}

Typical images that compared the influences of edaravone on the leukocyte rolling was shown in Figure 2. Image (a) was obtained in placebo group. Image (b) was obtained in edaravone group. The number of rolling leukocyte in edaravone group decreased significantly compared with placebo group.

\subsection{The Influence of Edaravone on the Leukocyte Rolling in DSS Induced Colitis Model}

The results that compared the influences of edaravone on the leukocyte rolling was shown in Figure 3. The number of leukocyte rolling in edaravone group decreased significantly compared with placebo group. The number of leukocyte rolling per 100 um vessel length for 10 minutes was $501.3 \pm 39.2$ in placebo group, $252.2 \pm$ 37.2 in edaravone group respectively (Figure 3 ).

\subsection{The Expression of P-Selectine on the Endothelial Cell}

The expression of P-selectine in the vascular endothe-

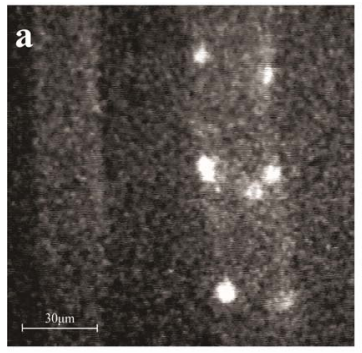

Placebo

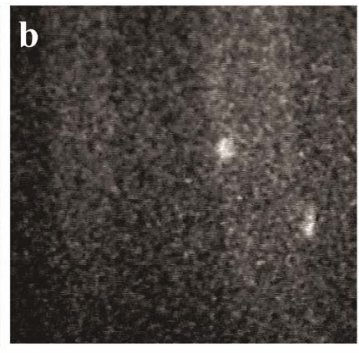

Edaravone
Figure 2. Imaging of leukocyte rolling with CFSE in colitis model (a) Placebo group; (b) Edaravone group $(10.5 \mathrm{mg} / \mathrm{kg} / \mathrm{hr}$ ); The diameter of vessel: $30 \mu \mathrm{m}$ (mesenteric artery).

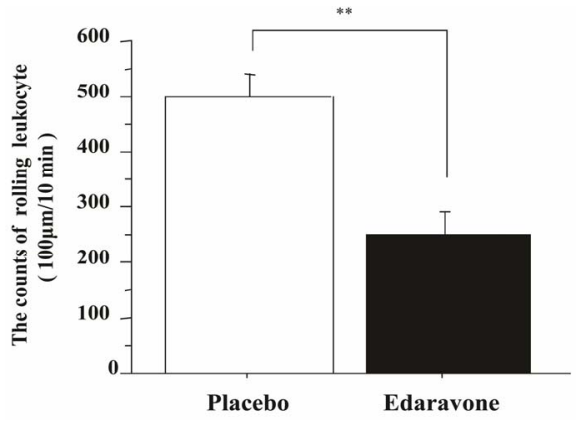

Figure 3. Comparison of rolling leukocytes in colitis model $\square$ : Placebo group; $\mathbf{\square}$ : Edaravone group $(10.5 \mathrm{mg} / \mathrm{kg} / \mathrm{hr}) ; \mathrm{n}=8,{ }^{* *}: \mathrm{p}<0.01$. 
lium in each group was examined by immunohistochemistry of cross-sections. Surface P-selectine was constantly observed regardless of inflammatory or non-inflammatory in placebo group, but not in edaravone group. Typical result in aortic root was shown in Figure $\mathbf{4}$ and, the same results were obtained at other area (aorta, superior mesenteric artery) (data not shown).

\subsection{The Expression of E-Selectine on the Endothelial Cell}

The expression of E-selectine in the vascular endothelium in each group was examined by immunohistochemistry of cross-sections. Surface E-selectine was not admitted in non-inflammatory model. While, in inflammatory model the expression of E-selectine was admitted in placebo group, but not in edaravone group. Typical result in aortic root was shown in Figure 5 and, the same results were obtained at other area (aorta, superior mesenteric artery) (data not shown).

\subsection{The Expression of ICAM-1 on the Endothelial Cell}

The expression of ICAM-1 in the vascular endothelium in each group was examined by immunohistochemistry of cross-sections. In aortic root and aorta, ICAM-1 expression in both groups was not admitted regardless of inflammation (data not shown). Similarly, in superior mesenteric artery, the expression of ICAM-1 was not admitted in non-inflammatory model (Figures 6(a) and (b)). However, the expression was admitted in placebo group of inflammatory model (Figure 6(c)). On the other hand, the expression was not admitted in edaravone group (Figure 6(d)).

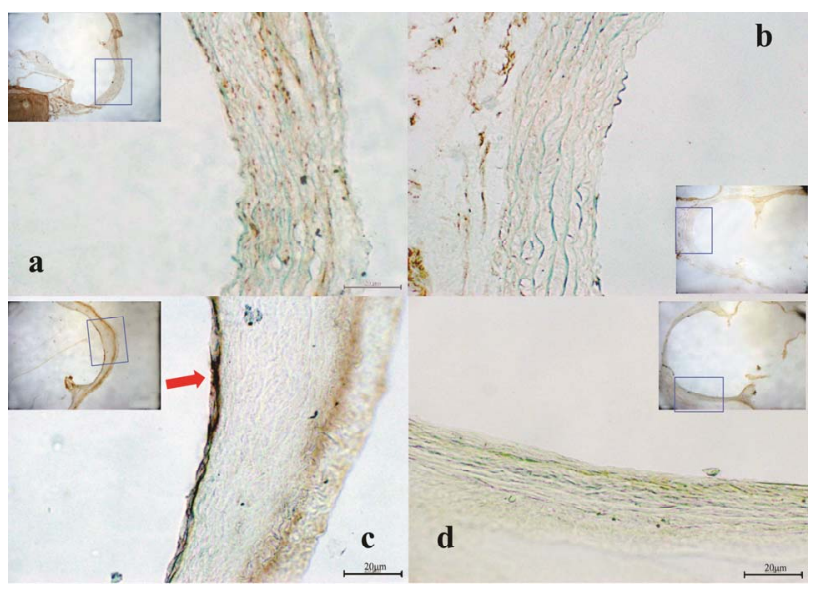

Figure 4. Immunohistologic findings in each group. (a) P-selectine staining in placebo group, (b) P-selectine staining in edaravone group $(10.5 \mathrm{mg} / \mathrm{kg} / \mathrm{hr})$, (c) P-selectine staining in DSS group, (d) P-selectine staining in DSS + edaravone group (10.5 mg/kg/hr); (a) (b): non-inflammatory model, (c) (d): inflammatory model, Magnification: $\times 20, \times 200$.

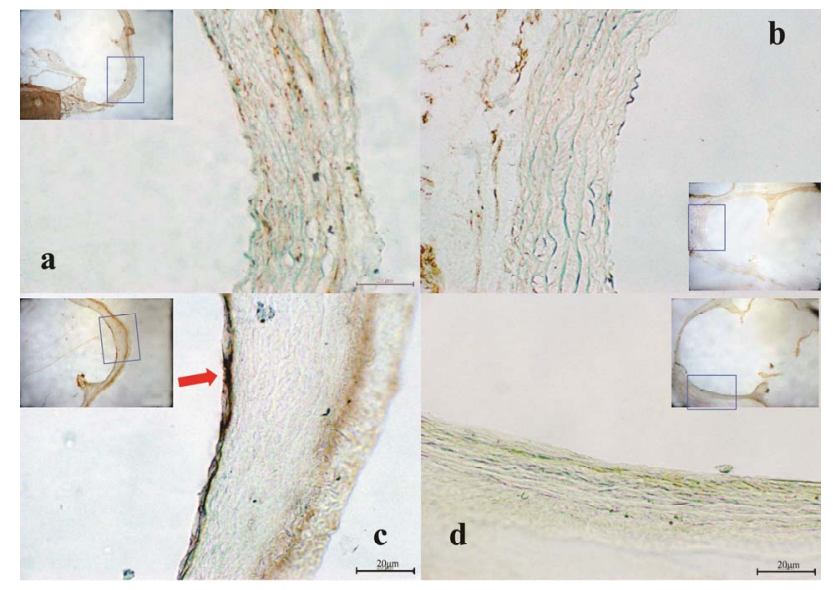

Figure 5. Immunohistologic findings in each group. (a) Eselectine staining in placebo group, (b) E-selectine staining in edaravone group $(10.5 \mathrm{mg} / \mathrm{kg} / \mathrm{hr})$, (c) E-selectine staining in DSS group, (d) E-selectine staining in DSS + edaravone group $(10.5 \mathrm{mg} / \mathrm{kg} / \mathrm{hr})$; (a) (b): non-inflammatory model, (c) (d): inflammatory model, Magnification: $\times 20, \times 200$.

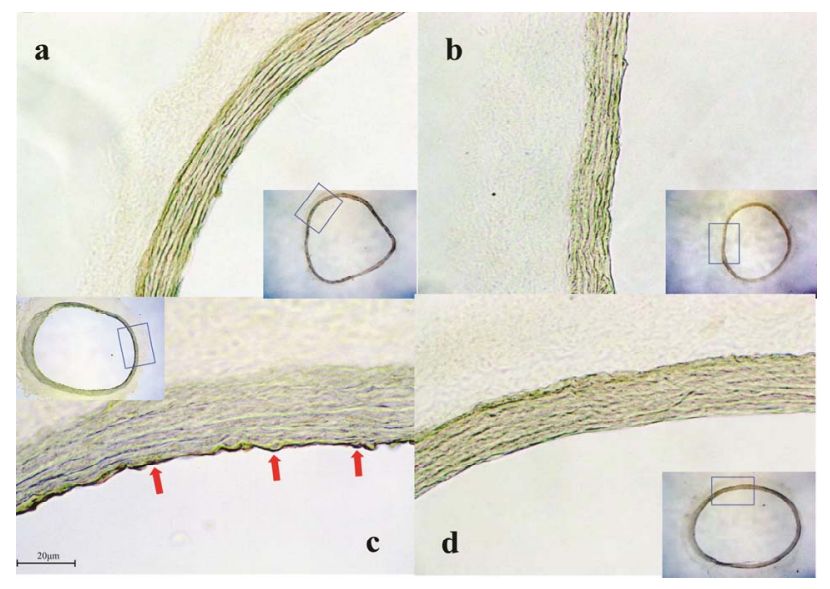

Figure 6. Immunohistologic findings in each group. (a) ICAM1 staining in placebo group, (b) ICAM-1staining in edaravone group (10.5 mg/kg/hr), (c) ICAM-1 staining in DSS group, (d) ICAM-1stainingin DSS + edaravone group $(10.5 \mathrm{mg} / \mathrm{kg} / \mathrm{hr})$; (a) (b): non-inflammatory model, (c) (d): inflammatory model, Magnification: $\times 20, \times 200$.

\section{DISCUSSION}

In the process to arteriosclerotic plaque formation, ROS and leukocytic dynamics are very critical. The present study examined the interaction of ROS and the leukokinetics from leukocyte rolling and the adhesion molecule expression with a radical scavenger. Especially, a lot of reports show that the oxidative damage of such a biogenic substance leads to the crises and development of atherosclerosis $[7,25,26]$. The leukocyte is one of the main ROS production sources in the site of inflammation. Some reports indicate that in vitro, the radical has working that improves the cell adhesion through inducing the expression of endothelial adhesion molecules (selectin 
and ICAM-1) [27], and an increasing of non-specific cell adhesion due to the damage of glycocalyx (glycocalyx) on the cell-surface [28] The present study examined the influence of edaravone on the leukocyte rolling and adhesion molecule expression that is the early response of leukocyte tissue-infiltrating by using in vivo model. The determination of the leukocyte rolling applied the biotechnology imaging model. The leukocyte was labeled by using CFSE as a fluorescent probe. CFSE, as a fluorescent probe, remains in the intracellular for a long time. The number of rolling leukocyte was based on the mensuration against a constant length of microvessel by real time confocal imaging in vivo.

The inflammatory condition based on increasing ROS production by accumulating leukocyte led to increasing the number of rolling leukocyte. The rolling leukocyte in inflammation model was significantly increased compared with non-inflammation model, and an increase in this leukocyte rolling was inhibited significantly by edaravone. So, the expression of selectin that play a key role for leukocyte rolling was examined. The cytokine stimulation by inflammation upregulated the expression of P-selectine. And this result supported the report that soluble P-selectin levels are critical as a risk factor for future vascular events [29]. P-selectin expression admitted at intact living endothelial cell, is an one evidence that P-selectin is necessary for microcirculatory homeostasis. On the other hand, this expression was significantly inhibited by edaravone. This result coincided with the imaging result that edaravone decreased rolling leukocytes in the bio-imaging model. The present study is primal report that experimentally showed that edaravone inhibits the P-selectin expression. These results suggest that ROS induces up-regulation of P-selectin expression. On the other hand, in non-inflammation model, the expression of E-selectin was hardly admitted in both groups at aortic root and aorta. The expression of E-selectin, unlike P-selectin, was not admitted in an intact endothelial cell. However, in inflammatory model, the expression of E-selectin was admitted in placebo group, and it suggested that up-regulation of E-selectin was induced by inflammation. These results are in agreement with the report that each mRNA of E-selectin express at the same time in the TNF- $\alpha$ induced inflammation model [30]. This expression was inhibited inedaravone group. It indicates that ROS has enhanced the expression of E-selectin as well as P-selectin. However, there is a report that TNF- $\alpha$ induced leukocyte rolling was not inhibited by E-selectin monoclonal antibody [30]. Therefore, a constant consensus is not yet obtained about the role of E-selectin in the leukocyte rolling. A further examination is necessary. These results suggest that the expression of these adhesion molecules is an enhancement mechanism of the leukocyte rolling by ROS. The leukocyte rolling phenomenon led to adhesion and infiltration [31]. In bioimaging model, a lot of leukocytes that adhered to the endothelial cell were admitted besides the rolling leukocyte. Then, the expression of ICAM-1 was examined as adhesion protein. The ICAM-1 manifestation is admitted by the vascular endothelial cell and the respiratory tract mucosa epithelial cell [32]. The ICAM-1 expression is usually little. However, the expression of ICAM-1 is enhanced by IL- 1 and TNF- $\alpha$ derived from macrophage and mast cell at inflammation [33,34].

In this condition, both groups did not admit the ICAM1 expression in both aortic root and aorta areas regardless of the inflammation or non-inflammation. On the other hand, the ICAM-1 expression was admitted in superior mesenteric artery. We speculated about the following possibilities as a one causative factor. The inflammation model used for the experiment is DSS induced colitis model. Therefore, the expression might have been admitted in regional blood vessel near from mesentery including inflammatory colon. This ICAM-1 expression was inhibited by edaravone. This suggests the possibility that the radical increases the expression of ICAM-1. A further examination is necessary for it whether the radical increases the expression of ICAM-1. However, it was speculated that the radical indirectly increased the ICAM-1 expression by inflammatory cytokine derived from endothelial cell injury [35] and accumulating leukocytes.

The present study showed that edaravone inhibited the expression induction of the adhesion factor by ROS. In addition, these results provide evidence for the inhibitory effect of edaravone against the risk factor of atherosclerotic development.

\section{REFERENCES}

[1] Jambunathan, N. (2010) Determination and detection of reactive oxygen species (ROS), lipid peroxidation, and electrolyte leakage in plants. Methods in Molecular Biology, 639, 292-298. doi:10.1007/978-1-60761-702-0 18

[2] Yamashita, T., Oda, E., Sano, T., Ijiru, Y., Giddings, J.C. and Yamamoto, J. (2005) Varying the ratio of dietary n-6/ n-3 polyunsaturated fatty acid alters the tendency to thrombosis and progress of atherosclerosis in apoE-/- LDLR-/double knockout mouse. Thrombosis Research, 116, 393401. doi:10.1016/j.thromres.2005.01.011

[3] Lu, M. and Gong, X. (2009) Upstream reactive oxidative species (ROS) signals in exogenous oxidative stress-induced mitochondrial dysfunction. Cell Biology International, 33, 658-664.

[4] Lau, A.T., Wang, Y. and Chiu, J.F. (2008) Reactive oxygen species: Current knowledge and applications in cancer research and therapeutic. Journal of Cellular Biochemistry, 104, 657-667.

[5] Ozono, R., Brydun, A., Watari, Y., Yamamoto, Y. and 
Ohshima, T. (2007) Assessment of oxidative stress in patients with atherosclerosis focusing on heme oxygenase. Rinsho Byori, 55, 758-763.

[6] Barry-Lane, P.A., Patterson, C., van der Merwe, M., Hu, Z., Holland, S.M., Yeh, E.T. and Runge, M.S. (2001) p47phox is required for atherosclerotic lesion progression in ApoE(-/-) mice. Journal of Clinical Investigation, 108, 1513-1522.

[7] Griendling, K.K., Sorescu, D., Lassegue, B. and UshioFukai, M. (2000) Modulation of protein kinase activity and gene expression by reactive oxygen species and their role in vascular physiology and pathophysiology. Arteriosclerosis, Thrombosis, and Vascular Biology, 20, 21752183.

[8] Ehara, S., Ueda, M., Naruko, T., Haze, K., Itoh, A., Otsuka, M., Komatsu, R., Matsuo, T., Itabe, H., Takano, T., Tsukamoto, Y., Yoshiyama, M., Takeuchi, K., Yoshikawa, J. and Becker, A.E. (2001) Elevated levels of oxidized low density lipoprotein show a positive relationship with the severity of acute coronary syndromes. Circulation, 103, 1955-1960.

[9] Meisinger, C., Baumert, J., Khuseyinova, N., Loewel, H. and Koenig, W. (2005) Plasma oxidized low-density lipoprotein, a strong predictor for acute coronary heart disease events in apparently healthy, middle-aged men from the general population. Circulation, 112, 651-657.

[10] Eriksson, E.E. (2003) Leukocyte recruitment to atherosclerotic lesions, a complex web of dynamic cellular and molecular interactions. Current Drug Targets-Cardiovascular \& Haematological Disorders, 3, 309-325.

[11] Eriksson, E.E. (2004) Mechanisms of leukocyte recruitment to atherosclerotic lesions: Future prospects. Current Opinion in Lipidology, 15, 553-558.

[12] Tanaka, M. (2002) Pharmacological and clinical profile of the free radical scavenger edaravone as a neuroprotective agent. Nihon Yakurigaku Zasshi, 119, 301-308.

[13] Xi, H., Akishita, M., Nagai, K., Yu, W., Hasegawa, H., Eto, M., Kozaki, K. and Toba, K. (2007) Potent free radical scavenger, edaravone, suppresses oxidative stress-induced endothelial damage and early atherosclerosis. Atherosclerosis, 191, 281-289.

[14] Group, E.A.I.S. (2003) Effect of a novel free radical scavenger, edaravone (MCI-186), on acute brain infarction. Randomized, placebo-controlled, double-blind study at multicenters. Cerebrovascular Diseases, 15, 222-229. doi:10.1159/000069318

[15] Watanabe, T., Tahara, M. and Todo, S. (2008) The novel antioxidant edaravone: From bench to bedside. Cardiovascular Therapeutics, 26, 101-114.

[16] Yoshida, H., Yanai, H., Namiki, Y., Fukatsu-Sasaki, K., Furutani, N. and Tada, N. (2006) Neuroprotective effects of edaravone: A novel free radical scavenger in cerebrovascular injury. CNS Drug Reviews, 12, 9-20.

[17] Zhang, N., Komine-Kobayashi, M., Tanaka, R., Liu, M., Mizuno, Y. and Urabe, T. (2005) Edaravone reduces early accumulation of oxidative products and sequential inflammatory responses after transient focal ischemia in mice brain. Stroke, 36, 2220-2225.

[18] Watanabe, T., Yuki, S., Egawa, M. and Nishi, H. (1994)
Protective effects of MCI-186 on cerebral ischemia: Possible involvement of free radical scavenging and antioxidant actions. Journal of Pharmacology and Experimental Therapeutics, 268, 1597-1604.

[19] Yoneda, Y., Uehara, T., Yamasaki, H., Kita, Y., Tabuchi, M. and Mori, E. (2003) Hospital-based study of the care and cost of acute ischemic stroke in Japan. Stroke, 34, 718-724.

[20] Barros, K.V., Xavier, R.A., Abreu, G.G., Martinez, C.A., Ribeiro, M.L., Gambero, A., Carvalho, P.O., Nascimento, C.M. and Silveira, V.L. (2010) Soybean and fish oil mixture increases IL-10, protects against DNA damage and decreases colonic inflammation in rats with dextran sulfate sodium (DSS) colitis. Lipids in Health and Disease, 9, 68. doi:10.1186/1476-511X-9-68

[21] Cooper, H.S., Murthy, S.N., Shah, R.S. and Sedergran, D.J. (1993) Clinicopathologic study of dextran sulfate sodium experimental murine colitis. Laboratory Investigation, 69, 238-249.

[22] Okayasu, I., Hatakeyama, S., Yamada, M., Ohkusa, T., Inagaki, Y. and Nakaya, R. (1990) A novel method in the induction of reliable experimental acute and chronic ulcerative colitis in mice. Gastroenterology, 98, 694-702.

[23] Seki, J. (1990) Fiber-optic laser-Doppler anemometer microscope developed for the measurement of microvascular red cell velocity. Microvascular Research, 40, 302-316.

[24] Teraki, Y., Moriya, N. and Shiohara, T. (1994) Drug-induced expression of intercellular adhesion molecule-1 on lesional keratinocytes in fixed drug eruption. American Journal of Pathology, 145, 550-560.

[25] Harrison, D., Griendling, K.K., Landmesser, U., Hornig, B. and Drexler, H. (2003) Role of oxidative stress in atherosclerosis. American Journal of Cardiology, 91, 7A-11A.

[26] Foncea, R., Carvajal, C., Almarza, C. and Leighton, F. (2000) Endothelial cell oxidative stress and signal transduction. Biological Research, 33, 89-96. doi:10.4067/S0716-97602000000200008

[27] Takano, M., Meneshian, A., Sheikh, E., Yamakawa, Y., Wilkins, K.B., Hopkins, E.A. and Bulkley, G.B. (2002) Rapid upregulation of endothelial P-selectin expression via reactive oxygen species generation. American Journal of Physiology-Heart and Circulatory Physiology, 283, H2054-H2061.

[28] Rubio-Gayosso, I., Platts, S.H. and Duling, B.R. (2006) Reactive oxygen species mediate modification of glycolcalyx during ischemia-reperfusion injury. American Journal of Physiology-Heart and Circulatory Physiology, 290, H2247-H2256.

[29] Ridker, P.M., Buring, J.E. and Rifai, N. (2001) Soluble Pselectin and the risk of future cardiovascular events. Circulation, 103, 491-495.

[30] Wan, M.X., Riaz, A.A., Schramm, R., Wang, Y., Vestweber, D., Menger, M.D. and Thorlacius, H. (2002) Leukocyte rolling is exclusively mediated by P-selectin in colonic venules. British Journal of Pharmacology, 135, 17491756.

[31] Ley, K. and Tedder, T.F. (1995) Leukocyte interactions with vascular endothelium. New insights into selectin- 
mediated attachment and rolling. The Journal of Immunology, 155, 525-528.

[32] Vainer, B., Horn, T. and Nielsen, O.H. (2006) Colonic epithelial cell expression of ICAM-1 relates to loss of surface continuity: A comparative study of inflammatory bowel disease and colonic neoplasms. Scandinavian Journal of Gastroenterology, 41, 318-325.

[33] Czech, W., Krutmann, J., Budnik, A., Schopf, E. and Kapp, A. (1993) Induction of intercellular adhesion molecule 1 (ICAM-1) expression in normal human eosinophils by inflammatory cytokines. Journal of Investigative Dermatology, 100, 417-423.

[34] Burke-Gaffney, A. and Hellewell, P.G. (1996) Tumour necrosis factor-alpha-induced ICAM-1 expression in human vascular endothelial and lung epithelial cells: Modulation by tyrosine kinase inhibitors. British Journal of Pharmacology, 119, 1149-1158.

[35] Hubbard, A.K. and Giardina, C. (2000) Regulation of ICAM-1 expression in mouse macrophages. Inflammation, 24, 115-125. 